\title{
Fiber Bragg Gratings in Microstructured Optical Fibers for Stress Monitoring
}

Geernaert/Thomas

Vrije Universiteit Brussel

Pleinlaan 2

1050 Brussel

\section{Fiber Bragg Gratings in Microstructured Optical Fibers for Stress Monitoring}

\author{
Thomas Geernaert ${ }^{\mathrm{a}}$, Geert Luyckx ${ }^{\mathrm{b}}$, Eli Voet ${ }^{\mathrm{b}}$, Tomasz Nasilowski ${ }^{\mathrm{a}}$, Karima Chah $^{\mathrm{a}}$, \\ Martin Becker ${ }^{c}$, Hartmut Bartelt ${ }^{c}$, Waclaw Urbanczyk ${ }^{\mathrm{d}}$, Jan Wojcik ${ }^{\mathrm{e}}$, Wim De Waele ${ }^{\mathrm{b}}$, \\ Joris Degrieck ${ }^{\mathrm{b}}$, Francis Berghmans ${ }^{\mathrm{a}}$, Hugo Thienpont ${ }^{\mathrm{a}}$ \\ ${ }^{a}$ Vrije Universiteit Brussel, Pleinlaan 2, 1050 Brussel, Belgium, email: tgeernae@vub.ac.be \\ ${ }^{b}$ Department of Materials Science and Engineering, Ghent University, 41 Sint-Pietersnieuwstraat, Gent 9000, \\ Belgium \\ ${ }^{c}$ Institute of Photonic Technology, Albert-Einstein-Strasse 9, D-07745 Jena, Germany \\ ${ }^{d}$ Wroclaw University of Technology, 27 Wybrzeze Wyspianskiego, 50-370 Wroclaw, Poland \\ ${ }^{\mathrm{e}}$ Maria Curie-Sklodowska University, PI. Marii Curie-Sklodowskiej 5, 20-031 Lublin, Poland
}

\begin{abstract}
Combining the functionalities of fiber Bragg gratings (FBG) and microstructured optical fibers (MOF) offers promising technological perspectives in the field of optical fiber sensors. Indeed, MOFs could overcome some of the limitations of FBGs in conventional fibers for sensor applications. The added value of MOFs stems from the ability to design an optical fiber in which an FBG acts as a sensor with a selective sensitivity, e.g. a sensor that is sensitive to directional strain but not to temperature. For this purpose we use a MOF with a phase modal birefringence on the order of $8 \times 10^{-3}$, which is more than the double obtained in conventional birefringent fibers. A FBG in this MOF results in two Bragg peak wavelengths, with a wavelength separation that depends on the phase modal birefringence. We characterize these FBGs for transversal loads on a bare fiber and compare the results with simulated sensitivities. Then, we embed the sensor in a composite coupon and we measure the response of the Bragg peak wavelengths as a function of the applied transversal pressure on the composite material. This allows drawing conclusions on the advantages of FBGs in MOFs for sensing applications.
\end{abstract}

\section{Introduction}

Fiber Bragg gratings (FBG) are widely recognized as one of today's most valuable optical fiber components in optical telecommunication, fiber laser technology and optical fiber sensing systems [1]. Measuring strain and structural damage are among the most important applications of FBG-based sensor technology. FBGs are commonly used to measure axial strain. However to monitor the structural health or assess damage of composite materials, it is also necessary to map the transversal stresses inside the material. For this purpose and as already reported, optical fibres with FBGs can be embedded in composite materials without compromising the structural integrity of the host material.

FBGs fabricated in microstructured optical fibers (MOF) have been reported in [2]-[7]. MOFs are a relatively new type of optical fiber that can be optimized for a large range of applications by tailoring the number, the size, the position and the geometry of the air holes that form the confining microstructure around a (sometimes doped) fiber core region [8]. Owing to this design flexibility, FBG-based sensors in MOFs promise optimized and selective sensitivities via dedicated air hole geometries.

Considerable effort is also invested in the polarization properties of MOFs. Highly birefringent ( $\mathrm{HiBi})$ MOFs are of interest because their birefringence (denoted by $B$ ) can be one order of magnitude larger 
than in standard $\mathrm{HiBi}$ fibers. In contrast to conventional polarization maintaining fibers, the temperature dependence of the birefringence in MOFs can be minimized (less than $10^{-4} \mathrm{~K}^{-1}$ ) compared to conventional polarization maintaining fibers, e.g. bow-tie HiBi fibers (about $-1 \mathrm{~K}^{-1}$ ). Therefore, PCFs have great potential to serve as selective sensors that do not require temperature compensation mechanisms.

In this report we study a FBG in a (highly birefringent) MOF to monitor transversal stress that is applied either to the bare fiber or when the FBG is embedded in a composite material. In birefringent fibers a FBG yields 2 reflection peaks with characteristic wavelengths $\lambda_{B 1}$ and $\lambda_{B 2}$, corresponding to the two orthogonally polarized fundamental modes propagating in the fibre (Eq. 1). These modes have an effective refractive index that depends on the mean effective refractive index $n_{\text {eff }}$ and the phase modal birefringence $B$. The measurement of the transversal stress that is applied to the composite sample is encoded in the wavelength separation $\Delta \lambda_{B}$ between the two FBG reflection peaks. This eliminates the need for absolute wavelength calibration and at the same time inherently provides correction for temperature variations.

$$
\begin{aligned}
& \lambda_{B 1}=2\left(n_{\text {eff }}+\frac{B}{2}\right) \Lambda \\
& \lambda_{B 2}=2\left(n_{\text {eff }}-\frac{B}{2}\right) \Lambda \\
& \Delta \lambda_{B}=\lambda_{B 2}-\lambda_{B 1}=2 B \Lambda
\end{aligned}
$$

After a few words on the FBG fabrication method, the remainder of this report first deals with finiteelement simulation results coupling both the mechanical and optical characteristics of the particular fiber geometry. The transversal line load sensitivity is derived from phase modal birefringence calculations under different transversal load orientations on the MOF. Then we compare these results with experimental data of the bare fiber characterization. Finally, we demonstrate stress sensing with the MOF embedded in a carbon fiber reinforced epoxy.

\section{Fabrication of FBGs in PCF}

So far, only a few reports on the mechanical sensitivities of FBGs in MOF have been published [9],[10]. The fabrication and characterization of FBGs in MOFs has nevertheless been dealt with several times [2][7]. Some publications have indicated that conventional FBG inscription setups are not always immediately suited for writing FBGs in MOFs [5],[11]. When the transverse laser writing beams propagate through the PCF microstructure, their wavefronts can be disturbed by the consecutive air-silica interfaces. As a result, it is not always possible to form a regular interference pattern in the core. This essentially depends on the characteristics of the MOF considered. Each microstructure will disturb the wavefronts of the laser writing beams in a different way, since its arrangement of airholes is unique. In addition, for each MOF, the orientation of the microstructure can also play an important role, since the geometry of the airholes can be asymmetric and thus variable towards the laser writing beams. The use of conventional UV inscription techniques is however still possible when the microstructure containts a (e.g. Germanium) doped core.

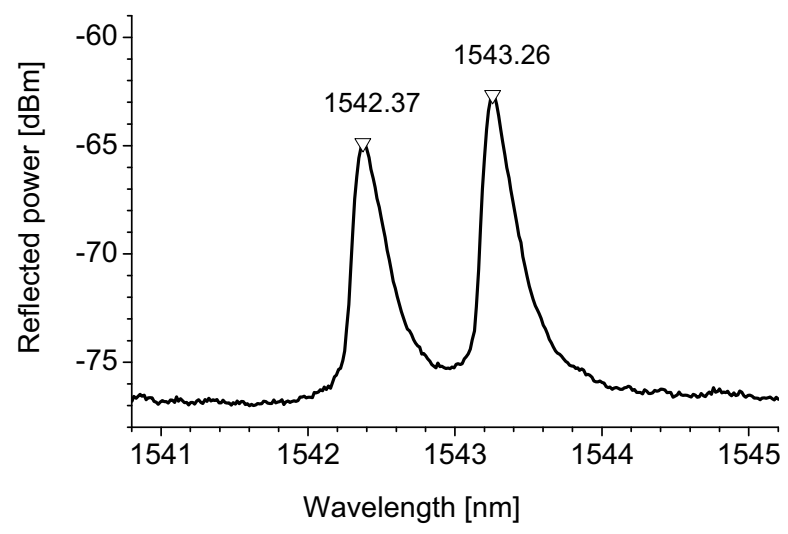

Figure 1: Reflection spectrum of a FBG in the reported highly birefringent PCF. The two Bragg peak wavelengths can be unambiguously extracted ftom this graph. 
Recently, we have reported on the fabrication of FBGs in hydrogenated Germanium-doped MOF with a frequency-doubled Argon laser in a conventional phase mask setup [12] and with an interferometric UV $\mathrm{KrF}$ excimer laser setup[13],[14]. The MOF used there is highly birefringent, which is of interest in sensing applications as previously mentioned, e.g. when the wavelength separation between the 2 FBG reflection peaks is considered as sensor signal. The reflection spectrum of the reported FBG is shown in Figure 1.

\section{Transversal loading of FBGs in birefringent MOF}

The MOF that we study here is a birefringent MOF that was previously reported in [15].The geometry of the microstructure is composed of three rows of airholes that surround a $7 \mathrm{~mol} \%$ Germanium doped central core. The microstructure of the MOF was modelled with COMSOL, a commercially available finite element software tool. The exact fibre cross-section topology was extracted from a SEM micrograph of fiber cross section. This approach allowed simulating both the mechanical and optical properties of the fiber sensor. In addition the results of the mechanical simulation can be easily exploited in the optical simulation of the fundamental modes and of their effective refractive indices.

When a transversal line load is applied to the fiber, the waveguide properties of the confining microstructure experience important changes: The microstructure can deform and the refractive index of the silica can become birefringent under influence of the induced material stresses.

The conversion of the material stresses into refractive index changes (Eq. 2) depends on the stress-optic coefficients $C_{1}$ and $C_{2}$ of silica [16]. The calculation of the mechanical variables requires the knowledge of the material parameters, which are relatively well-known for pure silica. The influence of Ge-doping on these parameters is less obvious, although several figures have been reported in literature [17].

$$
\left\{\begin{array}{l}
n_{x}=n_{x 0}+C_{1} \sigma_{x}+C_{2}\left(\sigma_{y}+\sigma_{z}\right) \\
n_{y}=n_{y 0}+C_{1} \sigma_{y}+C_{2}\left(\sigma_{x}+\sigma_{z}\right)
\end{array}\right.
$$

Because of the difference in thermal expansion coefficient and glass transition temperature between the undoped silica cladding and the doped core, there are already stresses present in the microstructure of the unloaded MOF after the fabrication process. The MOF is produced on a fiber drawing tower starting from a fiber preform that needs to be heated above the glass transition temperature of the used materials. During the drawing of the fiber, the silica cools down and stress appears in the initially unstressed regions due to their different thermo-mechanical properties. Because of this so-called thermal stress and due to the asymmetric nature of the microstructure, the phase modal birefringence in this fiber is inherently temperature dependent, as is the case for conventional birefringent optical fibers. However, the material birefringence in the core region is only responsible for a very small part of the overall phase modal birefringence, since this is primarily dominated by the asymmetric shape of the confining microstructure. The polarimetric temperature sensitivity of the MOF is therefore several orders of magnitude smaller than in conventional birefringent fibers.

The wavelength separation $\Delta \lambda_{B}$ between the two Bragg peak wavelengths depends on the phase modal birefringence $B$. Owing to the low polarimetric sensitivity of this MOF the wavelength separation also features an excellent temperature stability, which can be exploited to use this fiber for a temperature independent fiber optic sensor. We have simulated the change of $B$ for several orientations of a transversal line load on the bare fiber, to investigate the variation of the Bragg peak wavelength separation with the force orientation (Eq. 3). The graphs in Figure 2 show the distribution of the material birefringence in a cross section of the fiber and the microstructure. The geometrical arrangement of the airholes can induce local concentrations of higher or lower material birefringence in comparison with the rest of the fiber cladding. We have also verified this result experimentally with a setup that allowed applying a controlled line load up to $3 \mathrm{~N} / \mathrm{mm}$ onto two parallel plates between wich the sensor fiber and a dummy fiber were positioned under a controlled orientation. In Figure 3 both the simulated and experimental sensitivities are plotted versus the fiber orientation. Just as for conventional birefringent fibers, certain fiber orientations result in a positive change of the Bragg peak separation, and other orientations in a negative change. The sensitivity levels are also comparable with those of FBGs in e.g. bow-tie fiber [19]. 


$$
\frac{d\left(\Delta \lambda_{B}\right)}{d F}=2 \frac{d B}{d F} \Lambda
$$

The transversal line load sensitivity of FBGs in our MOF is comparable to that obtained in conventional birefringent fibers [19]. Depending on the fiber orientation, the sensitivity of the Bragg peak separation can be positive or negative with a maximum of about $100 \mathrm{pm} /(\mathrm{N} / \mathrm{mm})$. A FBG in this MOF has the advantage that the two Bragg peaks are clearly separated, which reduces the risk for overlapping and allows straigthforward detection of both Bragg peak wavelengths. In addition the temperature stability of the phase modal birefringence allows measuring the Bragg peak wavelengths without the need for a high absolute wavelength accuracy.

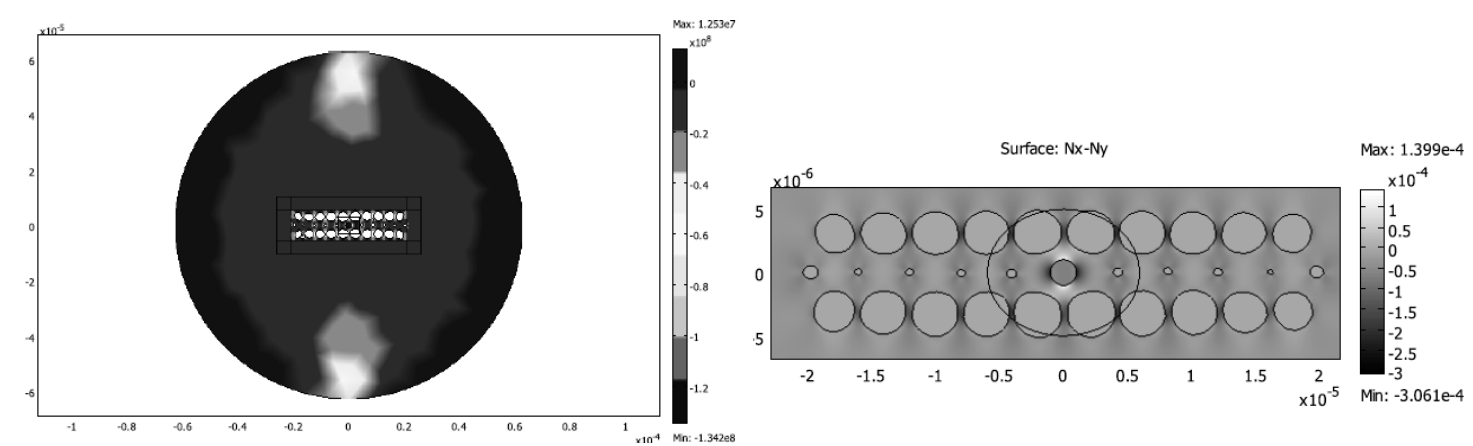

Figure 2: Left: A vertically applied line load induces a stress distribution in the fiber cross section. Right: The material birefringence in the microstructure (as a result of the stress distribution) depends on the precise airhole geometry and the material parameters of the doped and undoped regions.

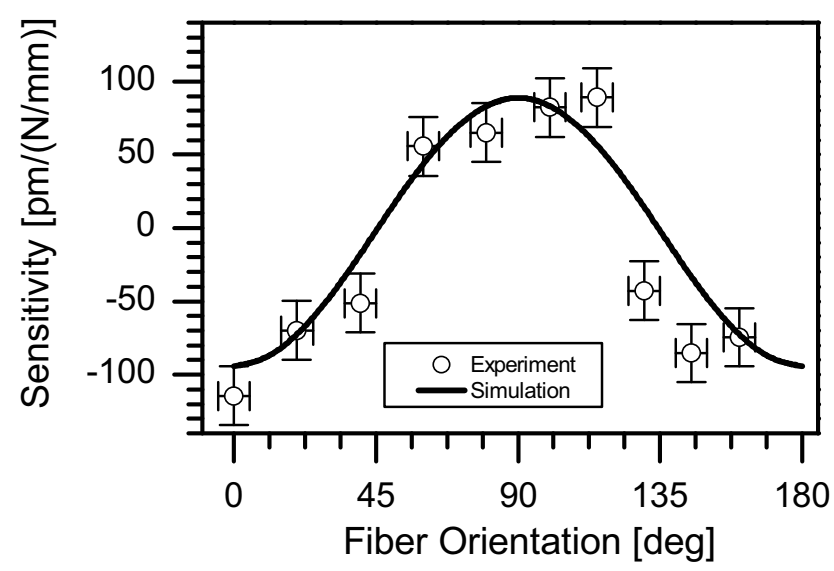

Figure 3: Experimental and simulated transversal line load sensitivity of the Bragg peak separation versus the fiber orientation. The line is the result of COMSOL simulations.

\section{Application in CFRP}

One of the important applications of transversal loads measurements can be found in structural health monitoring of carbon fiber reinforced composite (CFRP) material structures. CFRP materials feature a high strength and stiffness in the direction of the carbon fibers but are vulnerable to damage in the direction orthogonal to these fibres as there is only the much softer epoxy matrix that provides mechanical stability. Optical fibres have shown to be embeddable inside these materials, without compromising the structural integrity of the composite. Birefringent fibres with FBG sensors are promising candidates to detect structural damage to the material. The added value of using the above described MOF is its potential to lead to a FBG-based sensor that does not require additional temperature compensation schemes. We have therefore embedded our sensor inside a composite material. The fiber microstructure was oriented so that the transversal line load sensitivity was maximum, but negative. 
The FBG was more specifically embedded between the 2nd and the 3rd layer of a lay-up of 16 layers (thickness: approx. $0.1 \mathrm{~mm} / \mathrm{layer}$ ) carbon fiber epoxy laminate (M18/M55J material from Hexcel Inc.) The sample was cured with an autoclave under controlled pressure and temperature cycles. The final thickness of the sample was about $1.5 \mathrm{~mm}$. The mechanical tests were performed by mechanically loading the test samples with a universal testing machine. The transversal load was applied by two Tshaped iron profiles that were glued onto the composite coupon with epoxy glue. During the test, the transversal load in the composite material was gradually increased from 0 to 4MPa. The large contact area of the applied load $(4 \mathrm{~cm}$ by $9 \mathrm{~cm}$ ) assured a homogeneous distribution of the transversal load over the composite coupon. The FBG was located in the middle of the contact area. The Bragg peak wavelengths were simultaneously measured with an ASE-source (Amplified Spontaneous Emission) and a commercial FBG interrogator with a resolution of $1 \mathrm{pm}$. As can be seen in Figure 4, the Bragg peak separation was monotonously decreasing with increasing load. The linear change of the Bragg peak separation was measured to be $15.3 \mathrm{pm} / \mathrm{MPa}$. In a series of separate tests, we have confirmed that this sensitivity is comparable with the values for an FBG in bow-tie fiber.
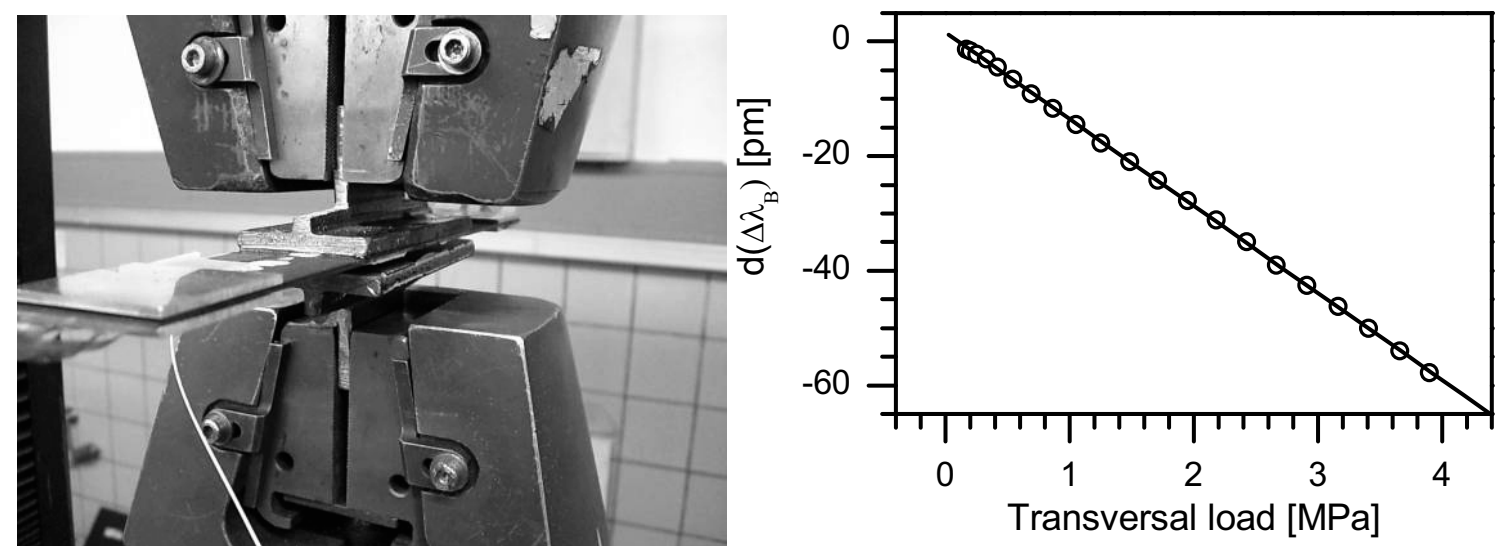

Figure 4: Left: Composite test sample in universal testing machine for transversal load testing. Right: Measured Bragg peak wavelength separation versus applied transversal load in the composite material. The line is a linear fit of the data points.

\section{Conclusions}

We have shown how the transversal line load sensitivity of a FBG in the studied MOF can be simulated with a finite element software tool. This transversal line load sensitivity of the Bragg peak wavelength separation was experimentally verified for different fiber orientations. In comparison with conventional birefringent fibers, the use of FBGs in birefringent MOFs benefits from the low polarimetric temperature sensitivity of these fibers. We have verified that FBG-based sensors can be embedded in composite materials for transversal stress sensing andfeature a linear response to the applied transversal oad.

\section{Acknowledgements}

Thomas Geernaert's research is funded by the Institute for the Promotion of Innovation through Science and Technology in Flanders (IWT-Vlaanderen). The work is supported in part by the EU FP6 Network of Excellence on Micro-Optics "NEMO", the EU FP7 project "PHOSFOS", the COST 299 action "FIDES" and the IWT SBO project "FAOS". Funding from the Research Foundation - Flanders (FWO-Vlaanderen), IAP and GOA is acknowledged as well.

Ghent University acknowledges the European Space Agency and FOS\&S for the use of the composite material provided by the MASSFOS project. The company FOS\&S is acknowledged as well for providing a commercial FBG interrogation system.

\section{References}

[1] A. Othonos and K. Kalli, "Fiber Bragg gratings: Fundamentals and Applications in Telecommunications and Sensing," Artech House, ISBN 0-89006-344-3, 1999

[2] B.J. Eggleton, P.S. Westbrook, R.S. Windeler, S. Spälter and T.A. Strasser, "Grating resonances in air-silica microstructured optical fibers," Opt. Lett. 24, 1460-1462 (1999). 
[3] N. Groothoff, J. Canning, E. Buckley, K. Lyttikainen, and J. Zagari, "Bragg gratings in air-silica structured fibers," Opt. Lett., vol. 28, pp. 233-235, 2003

[4] V. Beugin et al., "Efficient Bragg gratings in phosphosilicate and germanosilicate photonic crystal fiber," Appl. Opt., vol. 45, pp. 8186-8193, 2006

[5] J. Canning, N. Groothoff, K. Cook, C. Martelli, A. Pohl, J. Holdsworth, S. Bandyopadhyay and M. Stevenson, "Gratings in Structured Optical Fibres," Laser Chemistry, Vol. 2008, Article ID 239417

[6] M.C.P. Huy, G. Laffont, V. Dewynter, P. Ferdinand, P. Roy, J-L. Auguste, D. Pagnoux, W. Blanc, and B. Dussardier, "Three-hole microstructured optical fiber for efficient fiber Bragg grating refractometer," Opt. Lett. 32, 2390-2392 (2007)

[7] S.Pissadakis, M.Livitziis, G.Violakis and M.Konstantaki, Inscription of Bragg reflectors in all silica microstructured optical fibres using 248nm, picosecond and femtosecond laser radiation, Photonics Europe, Strasbourg, France, April 2008 (invited)

[8] P.S.J. Russell, "Photonic-Crystal Fibers", J. Lightwave Technol., vol. 24, pp. 4729-4749, 2006

[9] O. Frazao, J.P. Carvalho, L.A. Ferreira, F.M. Araújo and J.L. Santos, "Discrimination of strain and temperature using Bragg gratings in microstructured and standard optical fibres," Meas. Sci. Technol. 16, 2109-2113, (2005).

[10] C. Martelli, J. Canning, N. Groothoff, and K. Lyytikainen, "Strain and temperature characterization of photonic crystal fiber Bragg gratings," Opt. Lett. 30, 1785-1787 (2005)

[11] G.D. Marshall, D.J. Kan, A.A. Asatryan, L.C. Botten, and M.J. Withford, "Transverse coupling to the core of a photonic crystal fiber: the photo-inscription of gratings," Opt. Express 15, pp. 7876-7887 (2007).

[12] T. Geernaert, K. Chah, T. Nasilowski, H. Ottevaere, C. Caucheteur, P. Mégret, M. Szpulak, J. Olszewski, W. Urbanczyk, K. Poturaj, J. Wojcik, F. Berghmans and H. Thienpont, " Fiber Bragg Grating Inscription in Highly Asymmetric HiBi PCF with a Low Intensity UV CW laser, " Proceedings Symposium IEEE/LEOS Benelux Chapter, 2007, Brussels

[13] T. Geernaert, T. Nasilowski, K. Chah, M. Szpulak, J. Olszewski, G. Statkiewicz, J. Wojcik, K. Poturaj, W. Urbanczyk, M. Becker, M. Rothhardt, H. Bartelt, F. Berghmans and H. Thienpont, "Fiber Bragg Gratings in Germanium-Doped Highly Birefringent Microstructured Optical Fibers," IEEE Phot. Tech Lett., vol. 20, no. 8, pp. 554-556, 15 April, 2008

[14] T. Geernaert, T. Nasilowski, K. Chah, M. Becker, M. Rothhardt, M. Szpulak, J. Olszewski, K. Poturaj, J. Wojcik, W. Urbanczyk, H. Terryn, H. Bartelt, F. Berghmans and H. Thienpont, "The fabrication and characterization of fiber Bragg gratings in highly birefringent photonic crystal fibers for sensing applications," Proc. SPIE 6990, 69900C (2008), DOI:10.1117/12.781651

[15] T. Geernaert, G. Luyckx, E. Voet, T. Nasilowski, K. Chah, M. Becker, H. Bartelt, W. Urbanczyk, J. Wojcik, W. De Waele, J. Degrieck, H. Terryn, Francis Berghmans and H. Thienpont, " Transversal Load Sensing With Fiber Bragg Gratings in Microstructured Optical Fibers," IEEE Phot. Tech. Lett., vol. 21, no.8, pp.6-8, January 2009

[16] W. Primak and D. Post, "Photoelastic constants of vitreous silica and its elastic coefficient of refractive index, " J. Appl. Phys., vol. 30, pp. 779-788, 1959

[17] N. P. Bansal and R. H. Doremus, "Handbook of glass properties" (Academic Press, Inc., London, 1986)

[18] C.M. Lawrence et al., "Multi-Parameter Sensing with Fiber Bragg Gratings," Proc. SPIE, vol. 2872, pp. 24-31, 1996

[19] E. Chehura, C. C. Ye, S. E. Staines, S. W. James, and R. P. Tatam, "Characterization of the response of fibre Bragg gratings fabricated in stress and geometrically induced high birefringence fibres to temperature and transverse load, " Smart Mater. Structures, vol. 13, pp. 888-895, 2004. 